\title{
Educación Física para el desarrollo de la consciencia: Una propuesta pedagógica Physical Education for the development of consciousness: A pedagogical proposal
}

\author{
Cornelio Águila \\ Universidad de Almería (España)
}

\begin{abstract}
Resumen: En este ensayo se presenta una propuesta pedagógica en educación física dirigida a favorecer un aprendizaje significativo transferible a la vida cotidiana y una mayor consciencia social, a partir de la integración de mindfulness (consciencia plena) como base onto-epistemológica. Para ello, en la primera parte, se analiza el concepto de mindfulness desde su naturaleza esencial según la perspectiva budista, que integra sus fundamentos morales y sus implicaciones políticas. Asimismo, se critica el uso de mindfulness en el ámbito educativo como una mera técnica, para, a partir de ahí, defender las posibilidades de su inclusión como esencia onto-epistemológica de la acción pedagógica. En la segunda parte, se desarrollan los principios de esta propuesta de educación física enfocada a la expansión de la consciencia no condicionada del ser humano: una educación física orientada al crecimiento personal y moral, que estimule la participación política y contribuya a la transformación social.
\end{abstract}

Palabras clave: Mindfulness, corporeidad, educación física transformadora, ética, política.

\begin{abstract}
In this essay, a pedagogical proposal in physical education is presented aimed at promoting meaningful learning transferable to everyday life and greater social awareness, based on the integration of mindfulness as an onto-epistemological basis. For this, in the first part, the concept of mindfulness is analyzed from its essential nature according to the Buddhist perspective, which integrates its moral foundations and its political implications. Likewise, the use of mindfulness in the educational field is criticized as a mere technique, in order to, from there, defend the possibilities of its inclusion as an onto-epistemological essence of pedagogical action. In the second part, the principles of this proposal of physical education focused on the expansion of the unconditional consciousness of the human being are developed: a physical education oriented to personal and moral growth, which stimulates political participation and contributes to social transformation.
\end{abstract}

Keywords: Mindfulness, corporeality, transformative physical education, ethics, politics.

\section{La educación (física) a escena: retos y dilemas}

La educación hoy día se enfrenta a grandes retos y dilemas ante las importantes y continuas transformaciones que se están produciendo en todos los ámbitos personales y sociales. De acuerdo con Kirk (2008), los verdaderos retos están relacionados con la transferencia del aprendizaje a la vida, la significatividad y la autenticidad del conocimiento, y la contribución de las instituciones educativas a la transformación social. Sin embargo, los recientes cambios acaecidos en el ámbito educativo enfatizan su orientación instrumental en un sistema dominado por competencias enfocadas a la empleabilidad (Vicente, 2011), que podría tener efectos perjudiciales sobre la salud mental, emocional y social de niños/ as y jóvenes (Cigman, 2008). Ante este escenario, numerosos autores reivindican un cambio en el sistema que garantice la educación afectivo-emocional del alumnado, incorporando a los objetivos educativos rasgos como la confianza, la motivación, la resiliencia, el bienestar y la autoestima (Hyland, 2013). Ello implicaría re-enfocar la educación hacia el desarrollo personal y social, integrando las dimensiones cognitivas, afectivas, morales y espirituales por medio de procedimientos de acción pedagógica que estimulen el desarrollo de la consciencia de los participantes, tanto alumnado como profesorado (Gallifa, 2019).

La educación física (EF) no escapa de esta orientación instrumental al amparo de las competencias (MéndezAlonso et al., 2015; Vicente, 2011). La visión neoliberal de la educación refuerza la visión técnica mecanicista de la EF, sostenida por el discurso de rendimiento y la consideración

Fecha recepción: 24-06-19. Fecha de aceptación: 11-09-20

Cornelio Águila

cornelio@ual.es del cuerpo en su sentido biológico-mecánico, que oculta los rasgos subjetivos de la corporeidad (Fernández-Balboa \& Prados, 2012). Al respecto, en los últimos años, se han multiplicado las publicaciones sobre propuestas pedagógicas alternativas. Sin embargo, como plantea Vicente (2010), la mayor parte de estos intentos se centran en variaciones con un carácter eminentemente didáctico-técnico, exentos de fundamentos onto-epistemológicos sobre el sentido de nuestra materia y del cuerpo que promueve. Paralelamente, los también numerosos e interesantes ensayos que desde la teoría crítica se han formulado, salvo algunas excepciones (p.e. Vicente, 2016), suelen adolecer de falta de orientaciones prácticas. Este aparente divorcio entre pensamiento reflexivo y propuestas didácticas no ayuda a mejorar el valor de la EF, realzando múltiples incoherencias entre discurso y praxis: por un lado, la EF proclama el desarrollo de valores democráticos, el sentido crítico, la pluralidad y el respeto a la diversidad, mientras, por otro, su práctica reproduce las desigualdades sociales, las jerarquías, la homogeneización y el aprendizaje acrítico (Vicente, 2013).

Una EF que centre sus propuestas de cambio sólo en cuestiones técnicas, difícilmente podrá contribuir a estimular los potenciales del alumnado, a formar ciudadanos reflexivos, críticos y participativos, a respetar la diversidad cultural (Vicente, 2016), y a mejorar la convivencia en un marco ético y político sostenible. Así pues, la EF necesita un giro pedagógico para generar aprendizajes significativos (López et al., 2016), más allá de perspectivas que enfatizan lo físico y el discurso de rendimiento-competición (Valencia-Peris \& Lizandra, 2018). De acuerdo con Fernández-Balboa y Prados (2012), la EF requiere una profunda revisión de sus fines esenciales, superando las concepciones mecanicistas inspiradas en las ciencias físicas y biomédicas, para adoptar planteamientos filosóficos y pedagógicos centrados en desarro- 
llo de la consciencia del ser humano. En este sentido, en los últimos años vienen desarrollándose intervenciones basadas en mindfulness (MF) en el ámbito educativo que han mostrado su potencial como estrategia para la transformación personal y social aportando beneficios, tanto al alumnado como al profesorado, en los dominios cognitivo, afectivo e interpersonal (Palomero, 2016).

En este ensayo comparto una propuesta pedagógica para la EF desde un enfoque holístico - basada en mi experiencia docente universitaria - dirigida a atender las necesidades educativas formuladas por Kirk (2008). Esta experiencia se construye a partir de la integración de mindfulness como esencia onto-epistemológica. Para ello, inicialmente, reflexionaremos sobre el concepto mindfulness según su naturaleza original desde la perspectiva budista, su uso en educación, así como su integración en la práctica pedagógica de la EF. Posteriormente, se exponen las líneas para una EF enfocada al desarrollo de la consciencia del ser humano, que ayude al crecimiento personal y moral, estimule la participación política y contribuya a la transformación social.

\section{Mindfulness: bases onto-epistemológicas}

\section{Prefacio: la cosmovisión materialista y la enseñanza técnica}

La modernidad aportó un sistema de creencias basado en el paradigma científico materialista que, paulatinamente, fue impregnando nuestra idea sobre el mundo, de modo que está totalmente integrado en nuestra vida cotidiana, sin apenas ser conscientes de ello ni cuestionarlo (Taylor, 2018). El materialismo se basa en que la realidad primaria del universo es la materia y, por tanto, todo lo que existe tiene origen físico y se puede interpretar en términos físicos. Es el triunfo de la visión mecanicista sobre la vida, la naturaleza y el ser humano, que desestima interpretaciones metafísicas y reduce todo lo que se puede investigar y conocer a aquello observable y verificable, sentando las bases del positivismo lógico (Zohar \& Marshall, 2001). La realidad aparece atomizada, formada por distintos objetos separados entre sí (entre ellos el ser humano), identificando un mundo fuera de nosotros que puede ser comprendido, objetivamente, si lo observamos a distancia de modo que no «contaminemos» la realidad (Taylor, 2019). Este objetivismo legitima la visión cosificada dualista de la vida al enfrentar observador-observado, e implica un proceso por el que, lo vivido, queda transformado en lo pensado, definido, analizado y explicado, configurando un régimen de verdad (Foucault, 1992).

La cosmovisión materialista penetra todos los ámbitos, privados y públicos, por lo que incide tanto en la manera de construir nuestra identidad como en las formas de organizar lo social. Desde esta perspectiva, la razón científica fragmenta y cosifica la realidad y, en gran medida, obstaculiza la conexión entre las partes. Algunas consecuencias en el sistema educativo pueden observarse en la estructura de las distintas disciplinas académicas poco conectadas entre sí, o en el abandono de fines educativos globales no instrumentales. Aún más, la fragmentación alcanza a la concepción del ser humano que, compuesto por mente, cuerpo y emociones, rara vez es atendido en su globalidad en el sistema educativo. Así, la educación se convierte en un pro- ceso técnico de enseñanza de conocimientos legitimados por la ciencia positivista, basado en un realismo epistemológico objetivista, fragmentado y dualista (Spira, 2019). El sistema por competencias legitima y expande aún más este tipo de enseñanza, pues está construido a partir de un concepto de calidad basado en la racionalidad técnica (Vicente, 2010).

Esta perspectiva, prioriza el pensamiento sobre el cuerpo, negado y puesto al servicio de la mente (Águila \& López, 2019), una acción que es reforzada por la preeminencia de una EF técnica, que contempla el cuerpo como una máquina ajena a sus implicaciones éticas y políticas (Vicente, 2010). La hegemonía y la asunción acrítica de los postulados ontoepistemológicos del materialismo, dificulta el éxito de alternativas pedagógicas (Surdi \& Kunz, 2009), por lo que la educación (física) se mantiene como un mecanismo de estandarización basado en la estructuración de estrategias, objetivos y contenidos enfocados a obtener unos determinados resultados (Águila \& López, 2019; Méndez-Alonso et al, 2015).

Sin embargo, la ciencia tiene un alcance mucho mayor que el materialismo; hay otras formas de investigar y de ser racional desde perspectivas que aceptan la existencia de algo inmaterial, que es más esencial que la materia (Taylor, 2018). Para ello, sería necesario abrirnos a posiciones ontológicas no materialistas, sin perjuicio del rigor que se le presupone a la ciencia y a la educación. En este sentido, mindfulness responde a una ontología no materialista y no dualista más allá del dogmatismo científico hegemónico, considera al ser humano en su globalidad y la experiencia directa como base de la comprensión. De este modo, nos provee de fundamentos ontológicos y epistemológicos coherentes con una idea de EF crítica y transformadora de alto valor pedagógico.

¿De qué hablamos cuando hablamos de mindfulness?

De acuerdo con Davidson y Kaszniak (2015), podemos referimos a mindfulness en tres sentidos: estado de consciencia, práctica y/o rasgo de la persona. El término mindfulness (conocido en español como atención o consciencia plena) proviene de Sati de la antigua lengua pali, designa una actividad no conceptual y no lingüística basada en la experimentación directa, y hace referencia a tres aspectos: atención, consciencia y recuerdo (Miró, 2006). Sati es atención plena hacia un determinado soporte (respiración, objeto, sensaciones corporales...) que nos permite estabilizar la atención, emergiendo, así, una consciencia no condicionada que proviene de nuestro sentir puro directo y, al mismo tiempo, nos recuerda cuándo la atención se dispersa (Miró, 2006). Por tanto, MF es un estado de consciencia pura, no conceptual y no verbal, libre de juicios y análisis mental, conectado a la experiencia directa del presente, que disuelve las influencias del aprendizaje previo sobre la experiencia (Kabat-Zinn, 2009).

Para las principales tradiciones espirituales orientales, la esencia de la realidad no es la materia, sino una cualidad inmaterial o espiritual que impregna todo el universo (Taylor, 2018); una realidad ontológica pura no dual, una consciencia universal, que se manifiesta como materia y como mente, y se canaliza a través de todos los seres (Spira, 2019). El budismo utiliza el concepto de mente para referirse a la cons- 
ciencia, que es la base universal de la experiencia. Todos somos parte de una mente (consciencia) universal, y nuestra mente particular es creadora de la realidad que percibimos y, por tanto, de nuestra felicidad o sufrimiento. Según el monje budista Sogyal Rimpoché (2006), existen dos aspectos destacables de la mente: la mente ordinaria o sem, y la naturaleza misma de la mente, Rigpa.

La mente ordinaria responde a un flujo constante de pensamientos, emociones y sensaciones físicas, que nos sitúan en una consciencia dual. Ésta es la mente «que piensa, trama, desea, manipula, que monta en cólera, que crea oleadas de emociones y pensamientos negativos por los que se deja llevar» (Sogyal Rimpoché, 2006, p. 77). En este nivel de consciencia, nuestra identidad se basa en lo que pensamos y sentimos produciendo una imagen sobre nosotros mismos, el ego. El ego crea un marco de referencia a partir del cual percibimos y entendemos la realidad, es decir, funciona como un filtro de valoración e interpretación de las experiencias, de una manera automática puesto que es, en gran parte, inconsciente. Así, «la mente ordinaria es la presa incesante de las influencias exteriores, de las tendencias habituales y el condicionamiento» (Sogyal Rimpoché, 2006, p. 77), de modo que «la percepción de una imagen (o contenido) mental genera una reacción emocional (más o menos automática) que es positiva o negativa en función de si confirma o cuestiona el sentido establecido del ego» (Miró, 2006, p. 37).

La naturaleza misma de la mente, permanece «oculta en el interior de nuestra propia mente, nuestra sem, envuelta y oscurecida por el rápido discurrir de nuestros pensamientos y emociones» (Sogyal Rimpoché, 2006, p. 78). Para romper la identificación con el vínculo pensamiento-emoción del ego, «es necesario crear una situación de observación en la que la consciencia pueda percibir la imagen (o contenido) mental como algo distinto a la emoción que suscita» (Miró, 2006, p. 38). Así, el estado de MF nos acerca a una consciencia no condicionada que nos ayuda a «aliviar el sufrimiento y mejorar la convivencia, por medio del dominio de los estados aflictivos (ansiedad, depresión, desánimo, etc.) y del desarrollo de cualidades positivas, tales como la atención y el darse cuenta, la sabiduría, la compasión y la ecuanimidad» (Miró, 2006, p. 39). Y es aquí donde la práctica de MF va indisociablemente unida a sus fundamentos éticos y políticos. La base de una vida ética y política consciente incluye generosidad, confiabilidad, amabilidad, empatía, compasión, gratitud, alegría, inclusión y ecuanimidad, cualidades de la mente y del corazón que amplían las posibilidades de bienestar y claridad para uno mismo, y tienen efectos beneficiosos sobre el mundo (Hyland, 2013).

Nuestro estado mental no es ordinario o pleno en extremo, sino que está afectado por numerosos factores y es modificable a través de la práctica. La práctica de MF implica una acción intencional para alcanzar y mantener el estado de consciencia plena, lo que podemos entender, genéricamente, como meditación. En este sentido, y en base a mi experiencia personal, suelo diferenciar entre meditación formal e informal. El primer caso incluye aquellas prácticas específicas en las que se mantiene la atención plena hacia algún foco, y siguen una serie de indicaciones técnicas (v. Dahl, Lutz \& Davidson, 2015). Por su parte, la meditación informal conecta con la idea defendida por Kabat-Zinn (2009) de ex- pandir la consciencia plena a la vida cotidiana, es decir, encontrar un estado meditativo en cualquier cosa que estemos haciendo a través de nuestra presencia. Así, la práctica continuada de la meditación, tanto formal como informal, puede producir transformaciones estables en nuestro ser, emergiendo, entonces, MF como un rasgo de la persona. Para ello, necesitamos disciplina y perseverancia en la práctica, lo que nos puede acercar a un estado de «fluir atencional» de forma natural en el que se requiere menos esfuerzo (Ricard \& Singer, 2018).

La meditación y la práctica de MF son comunes en vías espirituales orientales como el hinduismo, el tantra o el taoísmo, si bien ha sido el budismo el que ha penetrado con más intensidad gracias, en gran medida, al desarrollo de un método que ha sido reconocido por la ciencia occidental (Cebolla \& Campos, 2016). Mindfulness proviene de la meditación en la atención plena -particularmente, la meditación vipassana- (Sayadaw, 2020) y, como defiende Kabat-Zinn (2009), uno de sus mayores impulsores, está orientada al desarrollo completo del ser humano, en sus dimensiones corporal, emocional, intelectual, espiritual y social. Esto implica que su significado original integra la práctica meditativa combinada con sus fundamentos morales y políticos (Williams \& Kabat-Zinn, 2011).

\section{Mindfulness en educación física: la experiencia cor- poral y motriz consciente}

El éxito de mindfulness en occidente está relacionado con la desvinculación de su sentido religioso y su aceptación en el ámbito académico y científico (Hyland, 2013). Ahora bien, esta popularización ha sido favorecida por el proceso de transformación que ha sufrido para adaptarse a la cultura occidental, pudiendo haberse convertido en un producto de consumo o en una tecnología de autocontrol (Águila, 2020a). En este sentido, Hyland (2017) denuncia la expansión de un MF superficial (McMindfulness) que se desarrolla a través de formas racionalizadas estandarizadas que impelen al consumo. Por su parte, Reveley (2016) muestra cómo MF, tal y como se está aplicando en el ámbito educativo, podría haberse convertido en una tecnología del yo que estimula un proceso de autocontrol y autorresponsabilidad en el alumnado orientado a la mejora del rendimiento y del bienestar propio, de acuerdo con la ideología neoliberal que necesita sujetos autónomos y emprendedores. Si bien la práctica de MF pudiera tener un componente técnico, tal y como señalé anteriormente respecto a la meditación formal, si es incorporado a la educación sólo como una mera técnica, enfocada al rendimiento, la estandarización y la medición de resultados, la meditación se convierte en una herramienta asequible, pero insustancial, al centrarse sólo en su forma sin considerar su fondo, esto es, sus fundamentos morales y las posibles consecuencias políticas (Águila, 2020b). Desde esta perspectiva, MF pierde su significado esencial, al verse distorsionado, explotado y extraído de su nicho ecológico (Williams \& Kabat-Zinn, 2011).

La integración de MF en educación física tiene el potencial de promover experiencias corporales y motrices conscientes, y estimular la reflexión crítica sobre las mismas, contemplando sus implicaciones éticas y políticas. En este sentido, el cuerpo es la puerta de entrada al estado de conscien- 
cia plena (Kabat-Zinn, 2009). Por ello, aunque inicialmente podría ser necesaria la práctica formal de la meditación para familiarizarse con el estado MF, la acción pedagógica de esta propuesta se orienta a promover este estado de consciencia en toda la práctica, llevando la atención a la experiencia, sin juicios, análisis, valoraciones o reacciones ante ella (Águila \& López, 2019). Muchas de las prácticas motrices orientales (p.e. yoga, chi-kung, aikido), así como diversos métodos de consciencia corporal occidentales (p.e. Feldenkrais, Alexander), se basan en la conexión cuerpo-mente-emociones, y pueden ser considerados ejercicios de meditación. Pero, esta propuesta no se basa en métodos específicos (aunque podrían utilizarse según las necesidades), sino en la práctica de MF en lo cotidiano, en mantener una actitud meditativa centrando la atención en aquello que estamos viviendo (Águila, 2020a). Por ello, cualquier situación pedagógica, en reposo o en movimiento, incluyendo el deporte tradicional (Torralba \& Santos, 2016), puede convertirse en un ejercicio de consciencia plena de manera intencional, sentando las bases de una EF que garantice el aprendizaje de movimientos significativos (Surdi \& Kunz, 2009).

\section{Educación física para el desarrollo de la consciencia}

Las bases esenciales de mindfulness que incluyen sus fundamentos morales e implicaciones políticas, nos brindan una oportunidad para revalorizar el potencial pedagógico de la EF, por lo que orientan esta propuesta en dos sentidos: por un lado, la perspectiva ontológica no materialista y no dualista de las tradiciones espirituales como el budismo, «descosifica» la realidad y reconoce su naturaleza unitaria (Taylor, 2018). Esto nos sitúa en un cuerpo vivo, que no es algo distinto a la mente, sino la misma consciencia que atraviesa la materia, y se manifiesta de una manera dinámica, en constante transformación (Mújica, 2020): una corporeidad como experiencia corporal impermanente que es la puerta hacia lo fenomenológico, y el vehículo de acceso a la consciencia plena (Sogyal Rimpoché, 2006). Por otro lado, los fundamentos éticos y políticos de la consciencia plena conectan con los principios humanistas universales, aportando herramientas que nos pueden ayudar a actuar en un sentido moral y estimular el compromiso social (Hyland, 2013), como desarrollaré posteriormente. Así, la inclusión de MF no se basa en su uso como una técnica puntual, sino en construir la relación pedagógica sobre la esencia de la consciencia plena. Para ello, es necesario centrarnos en la experiencia tal cual se está produciendo, sin atender al análisis mental de lo que implica nuestro estado de consciencia ordinario, el ego; es decir, el acceso a la esencia fenomenológica exige liberarnos de nuestros aprendizajes previos para centrar nuestra atención, intencionalmente, en el momento presente.

\section{Incitaciones pedagógicas}

La educación física para el desarrollo de la consciencia no condicionada del ser humano es un proceso que entrelaza el desarrollo personal -la estimulación de nuestro potencial y de las capacidades necesarias para disfrutar una vida sana, digna y feliz-, y una mayor consciencia social y compromiso político -saber cómo participar e influir en las activi- dades sociales- (Marina, 2006). Se basa en una ontología holística, no materialista (Taylor, 2018), reconociendo que la realidad es múltiple y subjetiva según los filtros que cada persona utiliza en su valoración del mundo, consecuencia de su aprendizaje vital corpóreo. Desde el punto de vista epistemológico, rechaza el dualismo, la fragmentación y el objetivismo del positivismo hegemónico. Por ello, la comprensión y la construcción del conocimiento se basan en la experiencia directa consciente (en la que todo nuestro ser está activo, mente, emocionalidad y cuerpo), y se obtienen a partir de la verdad que emerge de la unidad ontológica en la que la persona y el mundo son partes de una misma realidad (Singh Sikh \& Spence, 2016).

Esto nos lleva a un planteamiento metodológico que respeta la diversidad y la diferencia a través de aprendizajes de sujetos concretos (Vicente, 2016); que estimula la presencia activa del alumnado, la reflexión y la construcción personal del significado de la experiencia de una manera global, integrando las dimensiones cognitiva, emocional, corporal y social. El aprendizaje a través del estado de consciencia plena permite calmar nuestra mente ordinaria. Así, podemos mantenernos abiertos y receptivos a la información del presente, reduciendo el procesamiento auto-referencial y aumentando la consciencia corporal (Tang et al., 2015), siendo transferible a cualquier situación de la vida cotidiana (KabatZinn, 2009). Por tanto, la EF para el desarrollo de la consciencia se basa en la experiencia del ahora, en estimular la presencia de los participantes, presencia que «no consiste tanto en estar delante de [... ] cuanto en estar dentro, en contacto con uno mismo» (Toro, 2013, p. 302). Sin duda, aceptar el momento presente como base del aprendizaje requiere una disposición flexible en el profesorado respecto a contenidos y metodologías, y estar abierto a gestionar lo que acontece en cada situación: «un abordaje de las emociones, las actitudes y los valores no cuando toca el tema correspondiente [...] sino en las situaciones vivenciales concretas que naturalmente se dan en el aula» (Toro, 2013, p. 311). De este modo, la EF para el desarrollo de la consciencia se despliega a partir de los acontecimientos pedagógicos tal cual suceden, permitiendo que fluyan sin limitar la experiencia subjetiva. Esto requiere salvar los mecanismos de control que promueve la pedagogía por objetivos del sistema de competencias y abrirnos a principios de acción pedagógica que orientan nuestra labor (Touriñán, 2013), sin subyugar las experiencias tal y cómo las sienten los participantes.

Los fines de la EF, aquí, se orientan al desarrollo de una corporeidad consciente; a la expansión del rango de consciencia sensorio-corporal y motriz mediante propuestas que consideran la esencia del movimiento (y de la quietud) y no su forma estandarizada, estableciendo una relación reflexiva con el cuerpo (Surdi \& Kunz, 2009). El ser humano, entonces, es el sujeto protagonista de la experiencia, por lo que los contenidos deben superar las restricciones normativas para que estén al servicio de las necesidades reales del alumnado, que aporten aprendizajes corporales no reconocidos por la cultura hegemónica, así como sus representaciones éticas e implicaciones políticas (Vicente, 2016).

El principal soporte para acceder a la consciencia plena no condicionada es la respiración, que está asociada a la actividad mental (Kabat-Zinn, 2009). Llevar parte de la aten- 
ción a nuestra respiración y a nuestro cuerpo sentido nos conecta con el presente, nos abre hacia nuestro interior (Miró, 2006), por lo que es una herramienta básica en la estabilidad de la atención y la regulación emocional que debería aprenderse en EF. En este sentido, la importancia concedida a la competencia motriz en la actual ley de educación y la desaparición de contenidos relacionados con el cuerpo, como la consciencia y esquema corporal, en mi opinión, ha supuesto un empobrecimiento y una limitación del alcance de nuestra materia. Asimismo, el acceso a la consciencia plena requiere ralentizar el ritmo del trabajo, pausas y observación, incluso en las actividades más intensas (Águila \& López, 2019).

El aprendizaje holístico se construye a través del ciclo de la experiencia que integra las dimensiones intelectual, corporal, emocional y social, a través de diferentes momentos pedagógicos interconectados: 1) lecturas críticas sobre temas de interés; 2) experiencias corporales conscientes, centradas en la experimentación libre; 3) momentos para reflexionar sobre el sentir propio en relación con los aspectos conceptuales; y 4) espacios para compartir lo integrado con el resto de participantes. El diario de la experiencia es una herramienta importante que ayuda a integrar el aprendizaje. $\mathrm{Al}$ escribir sobre momentos de vida, al reflexionar sobre el proceso personal, se facilita escuchar aquello que silenciamos, ayudándonos a hacernos más conscientes y responsables de nuestras decisiones y acciones. Por otro lado, la evaluación es un proceso indisociable de la experiencia subjetiva: una toma de consciencia de la evolución y el desarrollo personal y del grupo, no definida por competencias motrices estandarizadas.

Desde esta perspectiva, el profesorado necesitaría desarrollar su propia corporeidad consciente para poder guiar al alumnado, es el recurso por excelencia (Toro, 2013). De este modo, la EF se concibe como un proceso para guiar al alumnado, para influir en él a través de la presencia del profesorado, lo que permite transmitir un mensaje (no necesariamente verbal) que integra coherentemente lo que sentimos, pensamos y hacemos (Águila \& López, 2019). Así pues, su función pasa por estimular y mantener la presencia de los participantes para crear un clima de confianza, de respeto a la individualidad y la diversidad, donde no hay juicios sino críticas constructivas que ayuden a las personas a expresarse tal como son; donde no hay estándares ni comparaciones, sino una experiencia intersubjetiva compartida, una relación de intercorporeidad.

La significatividad del conocimiento y la transferencia del aprendizaje a la vida: el desarrollo personal y moral

Mi cuerpo era una fortaleza multi-capa, una armadura gruesa, musculosa y fuerte [...] Ahora puedo ver el miedo que tenía hacia mi cuerpo, hacia lo que en verdad se esconde dentro. Yo solía proteger mi mente débil dentro de un cuerpo fuerte [... Cada vez que calmé mi cuerpo, cerré los ojos, respiré conscientemente y me relajé, sentí el llanto de una bestia dentro [...] Pensé que sólo al destruir el cuerpo, mi mente dejaría de oír esa bestia y volvería a ser yo mismo [... ] Puedo correr durante tres horas y media sin parar [...] no me importaba lo que hiciera o me doliera [... A Ahora [con el trabajo de consciencia corpo- ral] ya no siento la necesidad de destruir mi cuerpo [...] Mi armadura pasó de ser un frio mecanismo de defensa a convertirse en un aura radiante que emite calidez (Fernández-Balboa \& Prados, 2012, p. 13, original en inglés).

Este testimonio de un alumno universitario, muestra la relación moral que se establece con el propio cuerpo cuando es tratado como una máquina: una ética basada en el rendimiento y la productividad (Águila \& López, 2019). Refleja un uso funcional del cuerpo, al que se exige, pero no se cuida, ni se escucha cuando llora, grita, atrapado en la rigidez, en el miedo o la vergüenza (Prados, 2020). Pero, también apunta los potenciales del trabajo corporal consciente, y señala un camino de respeto y expansión de la corporeidad: «es como si mi cuerpo se hubiera convertido en una ventana que se abre a la vida, comunicando, liberándose [...] Puedo decir que he reforzado mi personalidad» (Fernández-Balboa \& Prados, 2012, p. 14).

La EF enfocada al desarrollo de la consciencia no establece orientaciones hacia un determinado tipo de cuerpo, ni potencia el aprendizaje de técnicas motrices estandarizadas. Al centrar la acción educativa en la persona y permitir la expresión consciente libre y subjetiva, favorecemos una relación moral amable y respetuosa con el propio cuerpo, contribuyendo al crecimiento personal (Fernández-Balboa \& Prados, 2012); creamos entornos de autonomía y de aprendizaje cooperativo (Bermejo et al., 2021), que estimulan la motivación intrínseca y la mejora del autoconcepto (MéndezGiménez et al., 2013). Para ello, es necesario convertir la corporeidad en el escenario de la interacción social bajo los principios del amor, el cuidado y la asistencia, hacia uno mismo y hacia los demás (García, 2017; Mújica \& Orellana, 2021).

La práctica de MF mejora la salud y el bienestar personal, con efectos positivos constatados científicamente sobre el estrés, la depresión, la ansiedad o el dolor crónico (Goleman \& Davison, 2017). Asimismo, se han demostrado mejoras a nivel emocional, psicosocial y en el desarrollo personal del alumnado de EF, al integrar en la práctica técnicas de meditación y yoga (Macías et al., 2019). Pero, además, puede contribuir a cambios positivos en la perspectiva del yo, gracias a una mejor regulación de la atención y de las emociones, y mayor consciencia corporal (Cebolla \& Campos, 2016). El proceso de auto-conocimiento a través de la consciencia plena permite identificar los patrones automáticos de nuestro ego para, distanciados de esa rígida identidad, poder explorar y ampliar nuestras posibilidades a través del cuerpo, mejorando la creatividad y la autonomía (Águila \& López, 2019).

En cuanto al desarrollo moral, la práctica de MF promueve la resiliencia, mejora la competencia emocional y, combinada con el cultivo de la empatía, la bondad y la compasión, apoya la acción constructiva y el comportamiento afectuoso (Salzberg \& Goldstein, 2001). Por ello, la centralidad de la dimensión ética de esta propuesta es evidente, por cuanto practicar MF se orienta hacia una ética que busca lo correcto en la comprensión, el pensamiento, la palabra, la acción, la ocupación, el esfuerzo, la atención y la concentración (Hyland, 2013). Esta ética de la virtud no se basa en principios abstractos universales, sino en la experiencia vivida 
conscientemente, y se orienta al desarrollo del carácter y la responsabilidad (Ricard, 2005). Esto implica reconocer los códigos morales occidentales como líneas orientadoras de nuestros actos que requieren flexibilidad y análisis de los contextos particulares, lo cual nos sitúa como sujetos con poder para decidir y asumir la responsabilidad de las consecuencias de nuestros actos. Actuar desde la aversión, la codicia o los celos, puede hacer que nos comportemos egoístamente. La presencia mental ecuánime del estado MF permite observar los condicionamientos de nuestro ego, ayuda a discernir las acciones que son positivas para el ser humano y para el mundo, capacidad denominada sabiduría discriminatoria (Salzberg \& Goldstein, 2001).

De este modo, el acto educativo estimula una presencia «que porque se da cuenta (es consciente) puede dar cuenta de lo que hace, desde dónde lo hace y cómo lo hace; una presencia consciente y ética» (Toro, 2013, p. 302). Esto dotaría al alumnado (y al profesorado) de herramientas aplicables a su vida cotidiana que le podrían ayudar a percibir sus prejuicios y condicionamientos, y, así, poder actuar de una manera más libre y ecuánime. Existen, pues, conexiones directas entre la claridad interior que nos permite ver el funcionamiento de nuestra mente, los fundamentos de la moralidad y la consciencia social (Gross, 2010). Como plantea Johnson (2010), no debemos olvidar que «la política no es más que la piel de la vida social bajo la cual encontramos una experiencia más profunda de nosotros mismos y los demás» (p. 37).

\section{Implicaciones políticas de la corporeidad consciente: la transformación social}

Educar la corporeidad consciente se basa en el respeto a la diferencia, la diversidad y la subjetividad, por lo que contribuye a la liberación del cuerpo funcional normativizado (Vicente, 2010). Trata al alumnado «como sujetos con poder y con capacidad para decidir sobre sus cuerpos [...] para participar, de acuerdo con una relación justa y no excluyente, en la toma de decisiones sobre lo individual y sobre lo colectivo» (Vicente, 2016, p. 81). Es un proceso de repolitización del cuerpo para, a partir de ahí, extenderse a otras problemáticas sociales. En este sentido, el trabajo de consciencia corporal nos brinda una experiencia en sí misma crítica, al desvelar los efectos de la normalización en el cuerpo. Valga, como ejemplo, el siguiente testimonio de un alumno universitario recogido por Prados (2020): «la consciencia corporal me ha hecho sentir la rigidez de mi cuerpo, ha sido como sentir la rigidez de los pupitres por los que he pasado» (p. 649). Esta toma de consciencia abre la posibilidad de experimentar procesos de liberación del cuerpo normativo, empoderamiento y reafirmación de nuestra corporeidad subjetiva (Águila \& López, 2019).

No obstante, esta propuesta pedagógica va más allá de las implicaciones políticas directas de lo corporal. Los críticos del capitalismo apuntan a la necesidad de regresar a valores colectivos más solidarios, a comunidades menos materialistas basadas en la confianza, la compasión y la empatía, un cambio que requiere una mayor atención a las emociones en la educación (Hyland, 2013). La EF que aquí defiendo, promueve el bienestar de los seres humanos y, al llevar la atención al sentir, la esfera crucial de las emociones humanas, puede contribuir decisivamente al desarrollo de valores que difícilmente pueden interiorizarse a través de medios puramente cognitivos (Águila, 2020; Águila \& López, 2019).

El materialismo legitima la visión dualista y cosificada de la realidad, construida según los patrones de la mente ordinaria, el ego. El ego, de acuerdo con Taylor (2018), nos crea un fuerte sentido de la individualidad y de separación dualista, que nos lleva a experimentar una sensación de aislamiento y carencia, y nos predispone a observar todo lo que «no soy yo» (la naturaleza, los otros...) como algo distinto y, en ocasiones, amenazante. Así, el materialismo «hizo más tolerable que nos comportáramos de manera egoísta y despiadada y menos aceptable que tuviéramos un comportamiento ético y compasivo [y] permite y alienta algunos de los peores aspectos de la naturaleza humana» (Taylor, 2018, p. 43) que tienen efectos negativos sobre el individuo, la sociedad y el medio ambiente.

Las respuestas emocionales automáticas de nuestro ego son obstáculos para el entendimiento y la buena convivencia, pues nos crean «miedos, frustraciones, incivismo y formas de dualismo» (Johnston, 2010, p. 35). La práctica de MF puede ayudar al control y transformación de las mismas para acercarnos a estados más serenos y ecuánimes. En este sentido, Reveley (2016) critica el uso de MF en educación, pues cree que pretende enseñar a los jóvenes a suprimir emociones negativas, como la ira, que son necesarias para el activismo político. Sin embargo, esta interpretación es errónea, pues «la práctica de MF no busca suprimir emociones, sino conocerlas, sostenerlas y transformarlas para que nuestras acciones sean constructivas, conscientes y no meros automatismos» (Águila, 2020a, p. 7). En este sentido, según el budismo Vajrayana, «cada emoción negativa posee cierta energía pura que puede ser liberada mediante la práctica. El enfado se transmuta en lucidez o, más exactamente, cuando las manifestaciones de enfado se calman, puede brillar la claridad subyacente» (Gross, 2010, p. 297). Por tanto, la consciencia plena nos aporta claridad y ecuanimidad, pues en la práctica meditativa encontramos en nuestras mentes «todas las fuerzas positivas y destructivas que se manifiestan en el mundo [de modo que] si queremos comprender el mundo, debemos comprendernos a nosotros mismos» (Goldstein, 2010, 155). Esto nos conecta con un poder reivindicativo, con una lucha contra todo lo que genera sufrimiento, pero de una manera no agresiva, ecuánime, compasiva y respetuosa. La siguiente conversación entre discípulo y maestro ilustra este argumento:

El enfado siempre es una pérdida de tiempo [...] Pero, ¿qué pasa con aquellas cosas que deben hacernos enfadar? Como los malos tratos [...] No te he dicho que abandonaras tu inteligencia crítica, te he dicho que el enfado es una pérdida de tiempo [... . Dominar el enfado mediante la renuncia a nuestra inteligencia crítica sería caer en la apatía y la ignorancia, y no alcanzar la realización (Gross, 2010, p. 299).

Situaciones sociales como la injusticia, la desigualdad, la guerra o la destrucción medioambiental, pueden provocar sentimientos de frustración y enfado. La práctica de MF aporta claridad a nuestra capacidad crítica, de modo que las emociones no determinen rígida y automáticamente nuestras acciones, como podemos colegir del proceso experimentado 
por la budista feminista Rita Gross:

La agresividad, el enfado y la aversión enmascaran la lucidez, pero la claridad está ahí de todos modos. Mis amigos me aseguraban erróneamente que a medida que mi práctica madurara, mi interés por el feminismo se desvanecería, pero lo que se desvaneció fue mi ira, haciendo más intensa y aguda la claridad de lo que ya había visto antes (Gross, 2010, p. 298).

El cambio empieza en uno mismo, pues la práctica continuada de MF aporta serenidad, compasión y un estado estable de presencia (Goldstein, 2010). Pero, dado que la realidad es una relación compartida, «en el mundo social y político [... ] sólo puede haber una reacción apropiada ante todos los seres vivos [...] la compasión y la bondad» (Johnson, 2010, p. 41). De este modo, la acción política en esta propuesta pedagógica, se centra en una manera de obrar e intervenir en sociedad de una manera consciente, ecuánime y compasiva, evitando el sufrimiento propio y de los demás. En este sentido, el estado de MF, al abrir un estado de consciencia que nos permite des-identificarnos con las respuestas automáticas de nuestro ego, nos ayuda a afrontar los conflictos en un sentido positivo, para convertirlos en una oportunidad de aprendizaje que ayuda a mejorar la convivencia y al desarrollo social (Carretero, 2017).

La consciencia plena conecta con los fundamentos morales de la humanidad en general, agregando, particularmente, dos elementos (Hyland, 2013): en primer lugar, la claridad de visión y la ecuánime estabilidad de cuerpo y mente que permiten la plena expresión de principios y de prácticas morales; y, en segundo lugar, la motivación para comprometerse con el mundo en la lucha contra la injusticia, la pobreza, la desigualdad y cuantos asuntos impidan el desarrollo del bienestar humano. De este modo, la EF para el desarrollo de la consciencia aboca a la participación política activa y al compromiso social en la vida cotidiana, pues la práctica continuada de MF nos conduce desde el egocentrismo hacia virtudes altruistas: el odio, la avaricia y el engaño son gradualmente reemplazados por la generosidad, la amabilidad y la comprensión sobre la naturaleza del mundo y la condición humana (Hyland, 2017). Pero, además, la EF debería incluir el cuestionamiento crítico de la ideología egoísta neoliberal (Vicente, 2013), la denuncia de situaciones de injusticia, codicia y degeneración social, así como la valoración de las desigualdades y conflictos que potencian el sufrimiento de los seres humanos.

\section{Comentarios finales}

En el análisis de los importantes dilemas globales de nuestro mundo, Harari (2018) señala el distanciamiento progresivo de nuestro cuerpo que el uso de las tecnologías está potenciando. A mi entender, los discursos hegemónicos sobre lo corporal y las orientaciones instrumentales de la EF también nos alejan de nuestra corporeidad. La separación de nuestro cuerpo, de los sentidos y del entorno en esta vida acelerada, consumista y egocéntrica que propone el capitalismo neoliberal, contribuye a nuestra desorientación y sufrimiento: difícilmente encontraremos la felicidad desconectados de nuestro cuerpo; difícilmente estaremos cómodos en el mundo, si no lo estamos en nuestro cuerpo.
Más que aprendizajes rígidos y estandarizados, el ser humano necesita aumentar la consciencia sobre sí mismo y sobre el mundo, encontrar el sentido de su propia vida y participar activamente en el devenir social. La EF tiene una gran oportunidad de elevar su estatus educativo puesto que es la única materia que, a priori, incide directamente en el cuerpo, siendo éste la vía de acceso a la consciencia plena. Confío, pues, que esta propuesta sea recibida como una optimista y constructiva llamada a la reflexión sobre las potenciales contribuciones de la EF para crear un mundo mejor.

\section{Referencias}

Águila, C. (2020a). Mindfulness y educación: ¿consumismo, tecnología o práctica transformadora? Espiral. Cuadernos del profesorado, 13(26), 1-11. Recuperado de http://ojs.ual.es/ojs/ index.php/ESPIRAL/article/view/2739/3363

Águila, C. (2020b). Mindfulness e investigación psicológica positivista: críticas y alternativas. Psychology, Society, \& Education, 12(1), 57-69. https://doi.org/10.25115/psye.v10i1.2159

Águila, C., \& López, J. J. (2019). Cuerpo, corporeidad y educación: una mirada reflexiva desde la Educación Física. Retos. Nuevas Tendencias en Educación Física, Deporte y Recreación, 35, 413-421. Recuperado de https://recyt.fecyt.es/ index.php/retos/article/view/62035/41748.

Bermejo, J. M. et al. (2021). Educación Física y universidad: Evaluación de una experiencia docente a través del aprendizaje cooperativo. Retos. Nuevas Tendencias en Educación Física, Deporte y Recreación, 39, 90-97. Recuperado de https:// recyt.fecyt.es/index.php/retos/article/view/77834/50075

Carretero, C. (2017). Mindfulness y educación emocional en la educación social: Reconstruirse para apoyar la reconstrucción del otro. Revista de Educación Social, 24, 1060-1068. Recuperado de http://eduso.net/res/wp-content/uploads/documentos/ 1032.pdf

Cebolla, A., \& Campos, D. (2016). Enseñar mindfulness: contextos de instrucción y pedagogía. Revista de Psicoterapia, 27(103), 103-118. https://doi.org/10.33898/rdp.v27i103.107

Cigman, R. (2008). Enhancing children. Journal of Philosophy of Education, 42, 539-557. https://doi.org/10.1111/j.14679752.2008.00648.x

Dahl, C. J., Lutz, A., \& Davidson, R. J. (2015). Reconstructing and deconstructing the self: cognitive mechanisms in meditation practice. Trends in Cognitive Sciences, 19(9), 515-523. http:// dx.doi.org/10.1016/j.tics.2015.07.001

Davidson, R. J., \& Kaszniak, A. (2015). Conceptual and Methodological Issues in Research on Mindfulness and Meditation. American Psychologist, 70(7), 581-592. https:// doi.org/10.1037/a0039512.

Fernández-Balboa, J. M., \& Prados, E. (2012). The conscious system for the movement technique: an ontological and holistic alternative for (Spanish) physical education in troubled times. Sport, Education and Society, 19(8), 1089-1106. https://doi.org/ 10.1080/13573322.2012.735652

Foucault, M. (1992). Microfísica del poder. Madrid: La Piqueta. Gallifa, J. (2019). Integral Thinking and is applicaton to Integral Education. Journal of International Education and Practice, 2(1), 15-25, 2019. https://doi.org/10.30564/jiep.v2i1.603

García, J. (2017). Los cuerpos van a la escuela, un favor que nos hace nuestro cerebro. Revista de Educación, 376, 9-32. https:/ /doi.org/10.4438/1988-592X-RE-2017-376-342

Goldstein, J. (2010). Tres métodos para lograr la paz. En McLeod, M. (ed.), Política con conciencia. La alternativa budista para hacer el mundo un lugar mejor (pp. 155-162). Barcelona: Kairós.

Goleman, D., \& Davidson, R. J. (2017). Los beneficios de la medi- 
tación. Barcelona: Kairós.

Gross, R. M. (2010). La sabiduría en la ira. En McLeod, M. (ed.), Política con conciencia. La alternativa budista para hacer el mundo un lugar mejor (pp. 289-305). Barcelona: Kairós.

Harari, Y. N. (2018). 21 lecciones para el siglo XXI. Barcelona: Debate (ed. Kindle).

Hyland, T. (2013). Moral Education, Mindfulness, and Social Engagement: Fostering Social Capital Through Therapeutic Buddhist Practice. SAGE Open, 1-9. Recuperado de https:// w w w.re se a r c h g a t e.net/public a tion/ 260291779_Moral_Education_Mindfulness_and_Social_Engagement_Fo stering_Social_Capital_Through_Therapeutic_Buddhist_Practice.

Hyland, T. (2017). McDonaldizing Spirituality: Mindfulness, Education and Consumerism. Journal of Transformative Education, 15(4), 334-356. https://doi.org/10.1177/ 1541344617696972

Johnson, C. R. (2010). Encarnar la paz. En McLeod, M. (ed.), Política con conciencia. La alternativa budista para hacer el mundo un lugar mejor (pp. 35-46). Barcelona: Kairós.

Kirk, D. (2008). Los futuros de la Educación Física: la importancia de la cultura física y la «idea de la idea» de Educación Física. En AAVV. Actas del V Congreso de la Asociación Española de Ciencias del Deporte. León: Universidad de León (CD-R).

Kabat-Zinn, J. (2009). Mindfulness en la vida cotidiana. Madrid: Paidós.

López, V., Pérez, D., Manrique, J.C., \& Monjas, R. (2016). Los retos de la Educación Física en el siglo XXI. Retos. Nuevas Tendencias en Educación Física, Deporte y Recreación, 29, 182-187. Recuperado de https://recyt.fecyt.es/index.php/retos/article/view/42552/25499

Marina, J. A. (2006). Aprender a convivir. Barcelona: Ariel.

Macías, A., García-García, J., Rossignoli, I., Valero-Valenzuela, A., \& Belando, N. (2019). Técnicas de autoconocimiento y autocontrol aplicadas en educación física para un mayor bienestar físico y mental en edades tempranas. Actividad física y Deporte: Ciencia y Profesión, 32, 54-68. Recuperado de http:/ /colefcafecv.com/wp-content/uploads/2020/02/ Rev_32_c_w.pdf

Méndez-Alonso, D., Fernández-Río, J., Méndez-Giménez, A., \& Prieto, J. A. (2015). Análisis de los currículos autonómicos LOMCE de Educación Física en Educación Primaria. Retos. Nuevas Tendencias en Educación Física, Deporte y Recreación, 28, 15-20. Recuperado de https://recyt.fecyt.es/index.php/ retos/article/view/34822/19057

Méndez-Giménez, A., Fernández-Río, J., \& Cecchini, J. A. (2013). Papel importante del alumnado, necesidades psicológicas básicas, regulaciones motivacionales y autoconcepto físico en educación física. Cuadernos de Psicología del Deporte, 13(1), 7182. Recuperado de https://revistas.um.es/cpd/article/view/ $177211 / 149041$.

Miró, M. T. (2006). La atención plena (mindfulness) como intervención clínica para aliviar el sufrimiento y mejorar la convivencia. Revista de psicoterapia, 17(66-67), 31-76.

Mújica, F. (2020). El término Educación Física en la postmodernidad: contribución de algunas perspectivas fenomenológicas. Retos. Nuevas tendencias en educación física, deporte y recreación, 38, 795-801 Recuperado de https:// recyt.fecyt.es/index.php/retos/article/view/7301/49514

Mújica, F., \& Orellana, N. (2021). El amor en educación física: una perspectiva filosófica basada en Max Sheler y José Ortega y Gasset. Retos. Nuevas tendencias en educación física, deporte y recreación, 39, Recuperado de https://recyt.fecyt.es/ index.php/retos/article/view/78787/50190

Palomero, J. E. (2016). Editorial. Mindfulness y educación. Revista Interuniversitaria de Formación del Profesorado, 87(30.3), 11-13. Recuperado de https://www.redalyc.org/jatsRepo/274/ 27449361004/html/index.html.

Prados, E. (2020). Pensar el cuerpo. De la expresión corporal a la conciencia expresivocorporal, un camino creativo narrativo en la formación inicial del profesorado. Retos. Nuevas tendencias en educación física, deporte y recreación, 37, 643-651. Recuperado de https://recyt.fecyt.es/index.php/retos/article/view/ 74256.

Reveley, J. (2016). Neoliberal meditations: How mindfulness training medicalizes education and responsibilizes young people. Policy Futures in Education, 14(4), 497-511. http://dx.doi.org/ 10.1177/1478210316637972

Ricard, M. (2005). En defensa de la felicidad. Barcelona: Urano

Ricard, M., \& Singer, W. (2018). Cerebro y meditación. Diálogo entre el budismo y las neurociencias. Barcelona: Kairós.

Salzberg, S., \& Goldstein, J. (2001). Insight meditation. Boulder: Sounds True.

Sayadaw, M. (2020). Mindfulness y vipassana. Barcelona: Kairós.

Singh Sikh, B., \& Spence, D. (2016). Methodology, Meditation, and Mindfulness: Toward a Mindfulness Hermeneutic. International Journal of Qualitative Methods, 15(1), 1-8. https:/ /doi.org/10.1177/1609406916641251

Sogyal Rimpoché (2006). El libro tibetano de la vida y la muerte. Barcelona: Urano.

Spira, R. (2019). La naturaleza de la conciencia. Barcelona: La Llave.

Surdi, A., \& Kunz, E. A (2009). Fenomenologia como Fundamentação para o Movimento Humano Significativo. Movimento, 15(2), 187-210. https://doi.org/10.22456/19828918.3054

Tang, Y. Y., Hölzel, B., \& Posner, M. (2015). The neuroscience of mindfulness meditation. Nature Reviews Neuroscience, 16, 213 225, 2015. http://dx.doi.org/10.1038/nrn3916

Taylor, S. (2018). Por una ciencia espiritual. Badalona: Koan.

Toro, J. M. (2013). ¿Metodologías de la interioridad? La presencia del maestro. En González, L.(ed). Maestros del Corazón. Hacia una pedagogía de la interioridad (pp. 295-327). Madrid: Wolters Kluwer.

Torralba, F., \& Santos, I. (2016). Inteligencia espiritual y deporte. Barcelona: Plataforma Editorial (ed. Kindle).

Touriñán, J. M. (2013). El significado de la función pedagógica y la necesidad de generar principio de acción. Revista Española de Pedagogía, 71(254), 29-47.

Valencia-Peris A. \& Lizandra, J. (2018). Cambios en la representación social de la educación física en la formación inicial del profesorado. Retos. Nuevas Tendencias en Educación Física, Deporte y Recreación, 34, 230-235. Recuperado de https:// recyt.fecyt.es/index.php/retos/article/view/60144/38606

Vicente, M. (2010). Educación Física e ideología. Creencias pedagógicas y dominación cultural en las enseñanzas escolares del cuerpo. Retos. Nuevas Tendencias en Educación Física, Deporte y Recreación, 17, 76-85. Recuperado de https:// recyt.fecyt.es/index.php/retos/article/view/34674/18785

Vicente, M. (2011). Escuela y educación física en el contexto de la enseñanza por competencias. Reflexiones genealógicas desde la pedagogía crítica. Cultura, Ciencia y Deporte, 6, 161-170. http://dx.doi.org/10.12800/ccd.v6i18.43.

Vicente, M. (2013). Crítica de la educación física y Educación Física Crítica en España. Estado (crítico) de la cuestión. Movimento, 19(1), 309-329. https://doi.org/10.22456/1982-8918.35661

Vicente, M. (2016). Bases para una didáctica crítica en educación física. Apunts. Educación Física y Deporte, 123, p. 76-82. https:/ /doi.org/10.5672/apunts.2014-0983.es.(2016/1).123.09

Williams, J. M. G., \& Kabat-Zinn, J. (2011). Mindfulness: Diverse perspectives on its meaning, origins and multiple applications at the intersection of science and dharma. Contemporary Buddhism, 12(1), 1-18. Recuperado de https:// w w w.tandfonline.com/doi/abs/10.1080/ 14639947.2011.564811.

Zohar, D., \& Marshall, I. (2001). Inteligencia espiritual. Barcelona: Plaza \& Janés. 